\title{
TV/Series
}

$1 \mid 2012$

Les Séries télévisées américaines contemporaines :

entre la fiction, les faits, et le réel

\section{Le Mormonisme selon Big Love : entre négativité et popularité}

Judith C. Warner

\section{OpenEdition}

Journals

Édition électronique

URL : http://journals.openedition.org/tvseries/1580

DOI : 10.4000/tvseries.1580

ISSN : 2266-0909

Éditeur

GRIC - Groupe de recherche Identités et Cultures

Référence électronique

Judith C. Warner, "Le Mormonisme selon Big Love : entre négativité et popularité », TV/Series [En

ligne], 1 | 2012, mis en ligne le 15 mai 2012, consulté le 19 avril 2019. URL : http://

journals.openedition.org/tvseries/1580; DOI : 10.4000/tvseries.1580

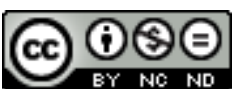

TV/Series est mis à disposition selon les termes de la licence Creative Commons Attribution - Pas d'Utilisation Commerciale - Pas de Modification 4.0 International. 


\title{
Le Mormonisme selon Big Love : entre négativité et popularité.
}

\author{
Judith C. WARNER
}

$B$ ig Love, série télévisée diffusée sur HBO du 12 mars 2006 au 20 mars 2011, met en scène une famille américaine mormone et polygame vivant dans l'Utah de nos jours. La famille se compose de Bill Henrickson, ses trois femmes Barb, Nicky et Margene, et de leurs huit enfants. Les Henrickson pourraient être de typiques Américains républicains de banlieue ( « suburb») si ce n'était pour leur mode de vie polygyne ${ }^{1}$.

Big Love peut être considérée comme une réinterprétation fictionnelle d'une histoire réelle : celle des Mormons de l'Utah. La série s'inspire librement de faits réels en y ajoutant la dose de fiction nécessaire à l'élaboration d'un programme télévisuel de divertissement. Le mormonisme ${ }^{2}$ est une religion fondée au $19^{\text {ème }}$ siècle aux États-Unis par Joseph Smith Jr. durant la période du Second Grand Réveil («Second Great Awakening»). La polygamie et le mormonisme sont respectivement une pratique et une religion marginalisées au sein de la société américaine, bien que le mormonisme soit représentatif d'une américanité culturelle certaine. Cette religion était peu représentée dans les médias, mais elle s'est peu à peu manifestée par des faits d'actualités notables. Ces faits, ajoutés à la réputation déjà peu clémente de la religion, ont permis d'amplifier les sentiments négatifs envers le mormonisme. Afin de comprendre comment la série - bien que très controversée elle aussi - a permis de

\footnotetext{
${ }^{1}$ Le terme polygynie est le terme exact définissant la pratique du mariage plural dans la religion mormone. Cependant les termes polygamies et polygynie seront employés de la même facon même si une différence existe entre ces deux termes. Ceci afin de suivre l'exemple américain, où les journaux et autres discours emploient le terme polygamie pour expliciter la pratique du mariage plural chez les Mormons fondamentalistes. De plus, le terme de polygamie est, en effet, le terme utilisé par la masse pour se référer à la polygynie qui consiste par définition à ce qu'un homme se marie avec plusieurs femmes conformément à une règle sociale, ou dans ce cas religieuse.

${ }^{2}$ Nous parlerons de « Mormonisme » pour se référer à la religion mormone, sa culture et ses principes, sans distinctions de dénominations. Ainsi le terme mormonisme englobera les diverses églises et interprétations contemporaines de la religion mormone (LDS, FLDS, etc.).
} 
populariser le mormonisme et d'alléger l'opinion stéréotypée, nous explorerons quelques échos entre fiction d'une part, et faits et personnages réels d'autre part. La série, en mettant en avant la doctrine mormone, n'a pas toujours contribué à l'amélioration de la représentation et de la perception de celle-ci ; néanmoins, elle a donné lieu à de nouvelles approches et réflexions sur le mormonisme moderne.

\section{Warren Jeffs / Juniper Creek}

Warren Jeffs, prophète et leader de l'Église Fondamentaliste de Jésus Christ des Saints des Derniers Jours (FLDS), a commencé à faire parler de lui en 2006 lorsqu'il a été officiellement placé sur la liste des fugitifs les plus recherchés des États-Unis, puis arrêté. S'en est suivi une bataille judiciaire qui continue encore aujourd'hui. La particularité des fondamentalistes est qu'ils pratiquent et revendiquent ouvertement le principe de mariage plural ${ }^{3}$. Big Love, de son côté, présente un mormon fondamentaliste du nom de Roman Grant, prophète auto-proclamé du « compound » de Juniper Creek, un lieu isolé de la ville où le prophète et sa (grande) famille vivent en réclusion, loin de la société contemporaine. Durant les saisons deux et trois (2007-2008), le personnage de Grant se voit pris dans une bataille judiciaire similaire à celle de Jeffs. Bien qu'il n'ait jamais été dit que le personnage de Grant est inspiré de Jeffs, la ressemblance dans l'attitude, les propos, l'histoire, voire le physique du personnage, se pose comme une évidence.

Big Love, à travers sa représentation des fondamentalistes, favorise l'enracinement de certaines opinions négatives. Les personnages d'Alby et Roman Grant et le procès fictif de ce dernier faisant directement écho à Warren Jeffs, son culte et son procès n'ont pas aidé à l'amélioration de l'image médiatique des mormons. L'idée qu'il existe encore aujourd'hui des mormons vivant en réclusion et pratiquant la polygamie est le point d'ancrage des diverses opinions et réactions véhémentes envers le mormonisme. Une personnalité telle que Warren Jeffs met - par ses actions, son image et sa réputation médiatique - l'accent sur l'aspect archaïque et abject de certaines pratiques du culte mormon (inceste, mariages forcés, mariages de mineurs, etc.). De telles images se font manifestes d'une réalité sans conteste: les soi-disant stéréotypes sont en fait fondés sur des faits avérés. Ils existent bel et bien des mormons pratiquant la polygamie et/ou vivant dans des « compounds ». Après des années de pratique et de prêche secrète du principe, et au vu des événements médiatiques de ces dernières années, il est donc légitime que la société américaine se

${ }^{3}$ Le principe du mariage plural est le terme officiel utilisé pour se référer à la pratique de la polygynie chez les Mormons. 
méfie 1) de la possibilité, devenue fait avéré, que la polygamie soit pratiquée illégalement par un grand nombre de mormons, 2) des déviances de la pratique de la polygamie chez les mormons. Du fait de cette généralisation, l'amalgame entre les mormons non-polygames et les fondamentalistes seraient le problème crucial conduisant à la stigmatisation du mormonisme : Big Love n'ayant pas aidé à rétablir une distinction claire. En effet, deux des personnages principaux, Nicky et Bill, sont issus du compound de Juniper Creek. Bien qu'ils en soient sortis, leur retour sporadique dans ce lieu ébranle la frontière entre ces deux modes de vie. La série rend la division entre le mormonisme "acceptable » et celui considéré comme archaïque, immoral et surtout illégal, confuse. Dans la série, cette frontière instable soutient l'idéologie de la réussite sociale sans dénigrement de ses racines. Cependant, elle se refuse à dénoncer des agissements et des coutumes marginalisés et dénigrés par le consensus américain et les autorités. Certaines institutions, la principale étant l'Église de Jésus Christ des Saints des Derniers Jours (Église LDS), tentent de contrer les effets néfastes du déballage médiatique autour d'événements tels que l'affaire Jeffs afin de minimiser les retombées négatives envers les mormons et le mormonisme en général.

\section{La « religion Big Love » et le mormonisme réel.}

Big Love représente le mormonisme en trois déclinaisons principales. On y retrouve les mormons fondamentalistes, les mormons de l'Église LDS, et une nouvelle forme de mormonisme incarné par la famille Henrickson, où le principe de mariage plural s'adapte à un mode de vie moderne. "Le mormonisme selon Big Love » est donc le miroir d'une réalité complexe et mal comprise. En effet, la série présente à la fois les principales branches du mormonisme telles qu'elles existent dans la réalité tout en bouleversant les limites entre chaque branche. On assiste ainsi à un va-et-vient des personnages entre les trois branches. Le triptyque religieux est complexifié à la fin de la série par l'introduction d'une nouvelle branche de la religion (une église mormone acceptant la prêtrise des femmes). Ce parti-pris de ne pas délimiter clairement les appartenances à l'une ou l'autre interprétation du mormonisme permet de contester le dualisme entre l'acceptable et le non-acceptable, le moral et l'immoral, ou encore entre la bonne et la mauvaise interprétation/pratique. L'idée de confusion se rapproche de l'idée de fragmentation d'un tout bien défini et unifié, illustré dans le générique de la série par le brisement de la glace entre les quatre personnages principaux. Cette image renvoie au discours tenu par les créateurs de la série qui défendent un modèle familial disruptif et atypique. Le choix du cadre du mormonisme et de la polygamie leur a permis de créer une histoire complexe explorant le thème d'un modèle familial non-traditionnel. Les créateurs, couple 
homosexuel à la ville, se servent de l'univers fictif pour faire passer un message, un appel à la tolérance et à l'acceptation des modes de vie singuliers. La série sert donc de décor à un message politique, social et engagé quant à la considération de la situation des homosexuels autant que des mormons au sein de la société américaine ${ }^{4}$. Cet engagement aura ainsi ouvert une brèche positive dans la représentation, la considération et la perception du mormonisme. Les mormons euxmêmes - avec l'exemple de Mitt Romney ou de l'émission Sister Wives de la chaîne TLC - ont donc profité de cette exposition pour mettre en lumière leur version de leur religion et de leur culture.

Malgré le message se voulant engagé et positif, la série - en n'établissant pas une division sans équivoque entre les différentes interprétations du mormonisme - nourrit l'amalgame qui laisse à penser que tous les mormons sont polygames ; amalgame contre lequel l'Église LDS se bat. Ainsi, l'Église LDS s'est farouchement opposée aux représentations faites dans la série en déclarant :

The central characters of Big Love are not "Mormons," or, more properly termed, Latter-day Saints. HBO has said the script makes it clear that members of The Church of Jesus Christ of Latter-day Saints don't practice polygamy. Still, placing the series in Salt Lake City, the international headquarters of The Church of Jesus Christ of Latterday Saints, is enough to blur the line between the modern Church and the program's subject matter and to reinforce old and long-outdated stereotypes ${ }^{5}$.

Dans ce même article, l'Église a entrepris de rappeler les différences entre les divers cultes communément appelés « Mormon », en insistant sur le fait que leur église - présentée comme l'église officielle désavouait le principe du mariage plural. Par conséquent, bien que la " religion Big Love » serve de couverture à un plus grand message, elle met en lumière la perception et la réputation du mormonisme.

$\mathrm{Au}$ vu des récents événements médiatiques impliquant des mormons, il semblerait que les médias soient plus enclins à aborder le sujet de la polygamie et du mormonisme de façon plus réfléchie et moins stéréotypée. Ce regain de popularité a permis à des mormons de sortir de l'ombre et de pouvoir aborder leur appartenance religieuse plus ouvertement, bien que l'affaire Jeffs, toujours d'actualité, soit là

${ }_{4}$ Ce fait a été confirmé par la déclaration de Tom Hanks, producteur exécutif de la série, sur la « Proposition 8 ». Hollie McKay, « Tom Hanks says Mormon Supporters of Proposition 8 are 'Un-American' », Fox News [en ligne], 16 janvier 2009 <http://www.foxnews.com/entertainment/2009/01/16/tom-hanks-says-mormonsupporters-proposition-american/> Consulté le 26 juillet 11.

5 The Church of Jesus Chrits of Latter Day Saints. « Church Responds to Question on HBO’s Big Love ", LDS Newsroom, <http://newsroom.lds.org/article/church-responds-toquestions-on-hbos-big-love> 06 mars 2006, Salt Lake City, consulté le 15 avril 11. 
pour rappeler les aspects néfastes de certaines interprétations du mormonisme.

La chaîne TLC diffuse pour la deuxième année consécutive une émission de télé-réalité sur une famille de mormons fondamentalistes: les Brown. L'émission Sister Wives ${ }^{6}$ suit la vie quotidienne de la famille au moment de l'arrivée d'une quatrième femme au sein du foyer. L'histoire se concentre sur les arrangements, jalousies, et autres complications que cette arrivée comporte. Un parallèle évident peut être établi entre l'émission de télé-réalité et la troisième saison de Big Love7. Durant ces deux saisons, les "sister wives » ont insistés sur la «normalité » de leur mode de vie, en comparant les problèmes et complications de leur couple à ceux d'un couple monogame ${ }^{8}$. Cette mise à nu de la famille Brown est bien sûr contrôlée et régie par la post-production. Un peu à la façon d'une série télévisée, l'émission présente une famille "glamourisée ", où il n'est guerre question de rentrer dans la vraie intimité des personnages. Car malgré l'appellation télé-réalité, force est de constater que les scènes sont choisies et montées afin de créer une atmosphère édulcorée, qui favorisera l'addiction du spectateur au programme. De plus, bien que l'émission soit centrée sur une famille de mormons polygames, la religion n'y est jamais mentionnée. Lorsque le mariage plural est évoqué, il l'est sous le nom de «style de vie» ou de «culture». Ce choix dans les termes n'est pas sans rappeler Joseph Smith Jr. qui présentait les mormons comme un peuple et une culture à part entière.

\section{Mitt Romney / Bill Henrickson}

Mitt Romney est un homme politique célèbre, candidat du parti républicain pour les prochaines élections présidentielles américaines de novembre 2012. Membre du parti républicain, il pourrait être qualifié de conservateur au vu de ses idéaux au sein du parti. Malgré la popularité de Romney pendant la campagne, ses détracteurs, une partie de l'opinion publique, et quelques journalistes se sont interrogés sur la place et l'influence de sa religion sur ses opinions politiques et sa campagne - Romney est membre de l'Église LDS. Romney n'a jamais caché son appartenance religieuse, cependant il a toujours affirmé que ses croyances n'interféraient en rien avec son implication dans la vie politique américaine et celle du parti 9 . En dépit

\footnotetext{
${ }^{6}$ « Sister wives » est le terme utilisé par les femmes qui composent un mariage plural pour parler de la « fratrie » qu'elles créent.

7 Dans la troisième saison de Big Love, Bill courtise puis épouse une quatrième femme.

${ }^{8}$ Meri: "Any polygamist marriage has ups and downs. Any monogamist marriage has ups and downs. It's just what happens." Sister Wives, TLC (2010- ).

9 Michael Luo, "Romney, Eye on Evangelicals, Defends His Faith", The New York Times, 7 décembre 2007, Téléchargé en ligne le 25 avril 2011.
} 
de cette affirmation, certains se sont demandés s'il était envisageable d'avoir un président mormon à la maison blanche ${ }^{10}$. L'émergence d'une telle question est probablement liée aux tribulations judiciaires de Warren Jeffs qui se déroulaient à la même période. L'affaire Jeffs a fait resurgir les questions et autres polémiques liées aux mystères de la doctrine mormone qui tournent essentiellement autour de la pratique de la polygamie. En effet, bien qu'abolie en 1890 par les autorités religieuses mormones, la continuité de la pratique du mariage plural par certains mormons reste une zone floue. Il a été démontré à plusieurs reprises via des affaires judiciaires, des témoignages autobiographiques et des enquêtes, que la polygamie était bel et bien encore pratiquée illégalement par un nombre inconnu de mormons. De plus Big Love, en représentant une famille polygame des temps modernes, n'a pas aidé à clarifier la situation.

La vie politique - bien que présente dans les deux dernières saisons de la série à travers la campagne puis l'élection de Bill à un poste de sénateur - semble avoir été épargnée par les diverses polémiques soulevées par la série. La représentation de la vie politique semble d'ailleurs n'avoir été utilisée que pour permettre le développement de l'histoire et des personnages autour de la vie publique d'une famille polygame. Le nom de Mitt Romney n'a été mentionné qu'une fois lors de l'épisode final de la série, et ce de façon très superficielle, évitant ainsi toutes conséquences ou débats (dans la série comme dans la réalité) autour de Mitt Romney lui-même et du rapprochement pouvant être fait entre Mitt Romney et le personnage de Bill Henrickson. Peut-être cela changera-t-il en 2012, avec la campagne présidentielle?

\section{Conclusion}

Big Love est une série marquante par le choix du type de famille qu'elle représente. Au moment où la communauté homosexuelle se bat pour faire reconnaître ses droits et accéder à l'institution du mariage de façon légale, la série arrivait à point nommé. Elle présente une conception du mariage et une dynamique de la famille différentes de celles d'un couple monogame hétérosexuel ${ }^{11}$. La représentation de la famille Henrickson et des divers aspects du

10 «A Mormon as President?» était le titre d'un article de Time centré sur ce questionnement de l'association entre mormonisme et politique à travers le personnage et la campagne de Mitt Romney. Mike Allen, "A Mormon as President? », Time, 26 novembre 2006.

${ }^{11}$ Bill Paxton, interviewé par Eric Spitznagel pour Vanity Fair, a déclaré: "I’ve always thought that the show is a metaphor for untraditional couples and untraditional families. We live in a modern society, where the traditional man-woman union isn't the only accepted norm anymore." "Bill Paxton Can Defend Polygamy, but He Can't Defend Sarah Palin », Vanity Fair [en ligne], 8 janvier 2010, Téléchargé le 26 mars 11. 
mormonisme a permis le traitement par les médias du sujet de la polygamie et du mormonisme sous un jour plus clément. La mise en parallèle de la version des Henrickson du mormonisme et de la polygamie et celle du compound de Juniper Creek ne peut être que favorable à la version Henrickson ainsi qu'à leurs homologues de la vie réelle. Que ce soit par le relatif succès de la série, la production puis diffusion de l'émission de télé réalité Sister Wives, ou même l'annonce de la candidature de Mitt Romney aux prochaines élections présidentielles, il est évident que la série a ouvert des voies pour que cette religion, cette culture se voit débarrassée, en partie, du stigmate dont elle est affublée depuis sa création.

La série met en scène une dialectique disruptive via la représentation de triptyques: le triptyque entre les trois branches principales du mormonisme; le triptyque créé par les 3 femmes de Bill et leurs différentes représentations de la construction féminine dans la société ; et enfin le triptyque de l'imbrication entre la vie de famille, la vie politique et la vie religieuse. Cette idée de triptyque, si l'on considère le message derrière la représentation du mormonisme selon Big Love, peut servir de rupture avec la définition consensuelle de la famille traditionnelle. La série offre une représentation de la famille américaine républicaine et conservatrice via le modèle d'une famille non-traditionnelle. Ainsi, elle tente de montrer que le modèle traditionnel de la famille peut être ré-envisagé sans pour autant mettre en péril les valeurs qui fondent la société américaine. 